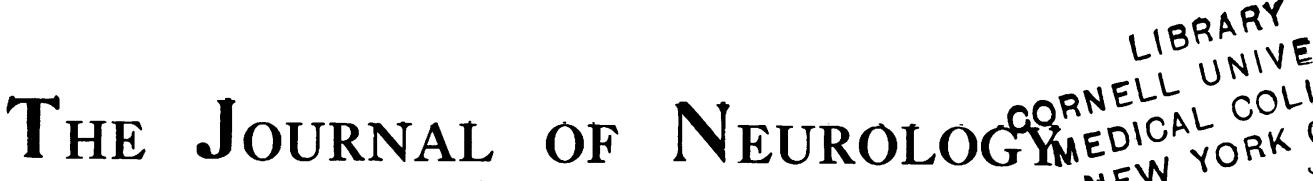

AND PSYChopathology

\begin{tabular}{lll}
\hline \hline Vol. XIII. & APRIL. 1933 & No. 52.
\end{tabular}

\section{Oríginal Dapers.}

\section{A FORM OF PROGRESSIVE GEREBRAL SGLEROSIS IN INFANTS ASSOGIATED WITH PRIMARY DE- GENERATION OF THE INTERFASGIGULAR GLIA.}

\section{Br}

\section{J. G. GREENFIELD, LoNDon.}

Progressive cerebral sclerosis in young children assumes two main forms for which the generic names of amaurotic family idiocy and progressive centrolobar sclerosis are perhaps the most suitable. The first has during the past 20 years largely yielded up its secrets to the histological investigations of a number of workers. The studies on neuronal metabolism in this disease by Marinesco, and of its Mendelian characteristics by Sjögren, deserve special mention in this connexion. Of the second form we know less, and the theories which have been advanced to account for it are largely speculative. Bielschowsky and Henneberg have divided cases of this kind into two main groups, the non-familial type of Schilder which they assume to be due to an exogenous infection, and a familial type which is attributed to an endogenous degenerative process. The latter has again been divided according to ageincidence into the infantile type of Krabbe, the juvenile type of Scholz and the slowly progressive type of Pelizaeus and Merzbacher. This division is somewhat arbitrary, since there is increasing evidence that many of the cases which have been described as Schilder's ' encephalitis periaxialis diffusa ' are familial. Thus Collier and Greenfield's case II appeared at first to be isolated, but three younger children of the family have since become affected on reaching a similar age; the post-mortem examination of the first two of these has revealed lesions in the brain which are almost identical with those described in the first child. It is noteworthy that in most families, though not in that of Symonds, the disease appears at the same age and runs a similar course in each of the members affected; it is no longer possible to describe a few main types, according to age of incidence and rapidity of involvement of the cerebral functions, seeing that the type varies with each affected family. 
From these familial cases which run a subacute course the form described by Pelizaeus and Merzbacher is fairly sharply delimited, owing to the extremely slow course of the disease and the peculiarly patchy destruction of the central white matter of the brain. I have had no opportunity of examining a case of this kind, and shall therefore confine discussion to subacute cases.

According to Bielschowsky and Henneberg the true cases of Schilder's encephalitis, that is those due to an exogenous infection, are differentiated from the familial degenerative cases by the presence of lymphocytic exudate round the vessels in the recently affected areas, and by the tendency of demyelinization to begin as perivascular zones which coalesce as they enlarge. (The very rapidly fatal case in an adult recorded by Stewart, Greenfield and Blandy in 1927 was of this nature.) In the degenerative and often familial type, they note that the process of myelin disintegration assumes a different form from that found either in Wallerian degeneration or in such acute demyelinating processes as disseminated sclerosis. Thus atypical pre-lipoids are formed which resemble in some ways those which fill the nerve-cells in amaurotic family idiocy, a point which has also been made by Coenen and Mir. They suggest that the origin of the trouble may lie in the circulation of abnormal lipoids in the blood, which are unsuitable for the formation or nutrition of myelin.

Collier and Greenfield (1924) suggested that Schilder's disease, in the familial case which they studied, was due to a primary affection of those neuroglial cells in the white matter which had to do with the nutrition of the myelin sheaths. The nutritive function of the oligodendroglia towards myelin was not recognised at this time, and in fact the first English description of oligodendroglia appeared in the same number of Brain.

Scholz considered the disease in his family to be a hereditary anomaly of the power of the neuroglia to regulate the metabolism of myelin. But he also pointed out that the myelin sheaths were most damaged in the regions of poorest blood supply.

Levaditi has recently propounded a somewhat different theory to account for the demyelination. He considers that it is due to a disturbance of lipoid metabolism on the part of the oligodendrocytes, so that instead of nourishing the myelin sheaths they remove the myelin from them. This inversion of the normal function of the oligodendrocytes is attributed to the direct action on them of a neurotropic virus which he terms ' oligodendrophile.'

Numerous workers, especially in America, have emphasized the fact that the oligodendrocytes tend to degenerate in the affected zones, usually going through the stage of acute swelling, and in many cases forming mucinoid material which with the disintegration of the cell-body escapes into the tissues. Eventually oligodendrocytes may completely disappear from the central parts of the degenerated areas; but, as far as can be gathered 
from descriptions and microphotographs, oligodendrocytes are always present in the less affected zones near the cortex.

It has occurred to some observers that the degeneration of oligodendrocytes may be independent of the demyelination, and may even be primary. Thus Del Rio Hortega (1927), being struck with the simultaneous disappearance of myelin and oligodendrocytes, was led to make the tentative hypothesis that oligodendrocytes might not only act as isolating and supporting structures but might also have to do with the nutrition of the myelin sheaths or the elaboration of myelin. This hypothesis has received some support from the work of Berliner, who observed that oligodendrocytes were present in the retina of the rabbit only in the areas where the nervefibres were myelinated. He suggested that the absence of a lamina cribosa in the rabbit permitted oligodendroblasts to migrate into the retina near the disc, and that they then formed myelin round the retinal nerve-fibres. These observations require corroboration. Before we can deduce from them a definitely myelogenetic function for the oligodendroglia, we must know whether opaque nerve-fibres can be present without oligodendrocytes, and vice versa. That the interfascicular glia has some nutritive action on the myelin sheath is however very generally accepted, and its homology with the cells of Schwann has been emphasized both by Del Rio Hortega and by others.

The two cases of progressive cerebral sclerosis here recorded, although they appear to differ in several respects from any cases of Schilder's disease which have been hitherto described, indicate the close association between demyelination and degeneration of the interfascicular oligodendrocytes. They are singular in that the disease has affected tracts and fibre-systems rather than contiguous areas, and in that the oligodendroglia has degenerated and disappeared from fibre-systems which are still fairly well myelinated.

\section{PERSONAL GASES.}

CASE I. B. R. (female), age 3, was admitted to the National Hospital, Queen Square, under Dr. Critchley, on January 12, 1932, and died on January 16. She was the only child of healthy parents, born at full term and weighing then $7 \frac{1}{2}$ lbs. Pregnancy had been normal except that the mother had felt no movements until one week before her confinement. Labour was normal. The child had been lively and vigorous as an infant. She walked when 11 months old and talked at 16 months. When 18 months old she developed a squint which her mother attributed to a fall on her forehead. This was cured by glasses in six months. At the age of 22 months she had whooping-cough. At the age of two she began to fall backwards when walking, at first only two or three times a day, but progressively more often, so that in six weeks she could only walk when she held her mother's hand. By the time she was two and a quarter she could only stand when holding a chair, and when she was two and a half she could do nothing but lie and had lost the use of her hands. The mother stated that there had been no staggering or unsteadiness, but simply progressive loss of strength. The child had also complained of a sensation of 
pins and needles at first in her legs and later in her arms, which lasted for three months from the age of two and a half years. At this time she was quite bright, could see and speak normally and could move her head. She was sent first to an orthopædic hospital in Birmingham and after a few days transferred to Leicester Royal Infirmary. When there she changed rapidly, becoming speechless and senseless; she did not know her mother, but turned her head continually from side to side as though it hurt her. After about eight weeks she appeared rather brighter, so that she knew her mother and father, but she seemed to have pain in her limbs. She appeared to be able to see at this time. During the last three months before admission to the National Hospital her eyesight had failed gradually and for the last four weeks she had been blind. She was still able to swallow but did not chew her food. There had been no convulsions at any time.

On examination in the National Hospital she was seen to be a fairly well nourished child of the normal size for her age. She lay in bed with the head retracted, the arms extended, the back bent forwards and the legs crossed. She whined continually. She was quite incooperative and inattentive, but she opened her eyes widely if her name was spoken in either ear. She seemed to be quite blind. The skull measured $49.5 \mathrm{~cm}$. in its greatest circumference. The pupils reacted to light but constantly varied in size. The optic discs and retinæ appeared normal. The corneal reflexes were absent, but pinprick was responded to over the face and body by increased crying. The limbs tended to be held rigidly, especially if handled. The arms were held extended at the elbow, pronated with the fingers tightly closed. The legs were somewhat flexed at the hip, and extended at the knee, the left being crossed over the right. The feet were inverted. Passive movements of the head caused alterations in the position of the limbs to some extent. The deep and superficial reflexes were completely suppressed with the exception of the plantars. Here stimulation of the soles of the feet produced fanning of the tones on both sides, with little dorsiflexion of the great toe.

She died from bronchitis on the fourth day after admission to the hospital.

\section{PATHOLOGY.}

The post-mortem examination was delayed for 44 hours after death and the brain only was examined. The dura mater was slightly adherent to the skull. The brain appeared congested but otherwise its external surface looked normal. It was soft but not abnormally so. The occipital convolutions were small and numerous, but there was no obvious shrinkage of this part of the brain. The corpus callosum tore easily. On making horizontal sections through the brain the white matter appeared rather greyer than normal and the centrum ovale was much narrower than in Case II. In the occipital lobes the subcortical fibres looked rather whiter than the deeper zones of the centrum ovale, but the difference was slight. The optic thalamus was shrunken, pale and firm, and the internal capsule was softened in its inner zone and had split away from the optic thalamus and the body of the caudate nucleus. The island of Reil was sunk rather deep beneath its opercula, apparently owing to the shrinkage of the optic thalamus.

Sections stained for myelin by the Wolter (Kulschitsky-Pal) method showed a widespread deficiency of myelinated fibres, most evident in the 
more posterior (occipital) parts of the white matter, while the optic radiations, which were relatively intact, showed up as a dark band. There was however some loss here also and many of the myelin sheaths were beaded or locally swollen. In the frontal lobes many fine myelin sheaths were visible under the microscope, although the general appearance to the naked eye was that of complete demyelination. There was no sparing of the subcortical zone, but some myelinated fibres were present in the white centre of a few of the convolutions, and radial fibres and a few tangential fibres were seen

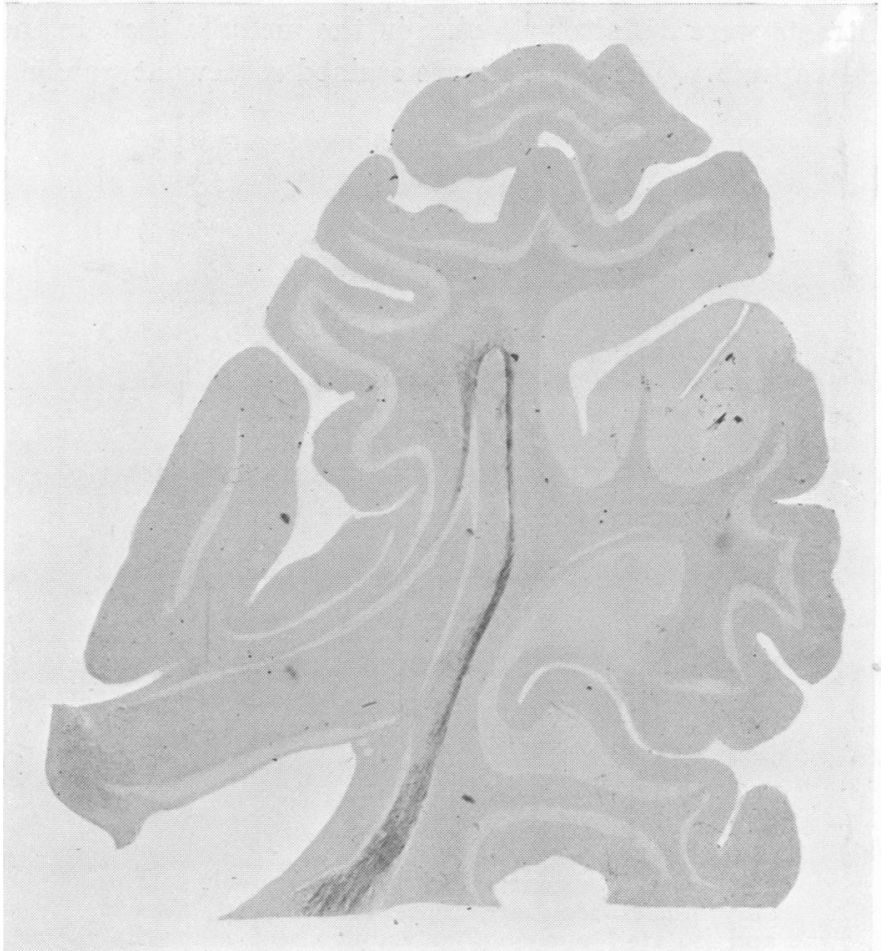

Fig. 1. Horizontal section of the occipital lobe, stained by Weigert-Pal method, showing the general absence of myelinated fibres except in the optic radiations, which stain fairly dark.

in the deeper layers of the cortex in many places. The internal capsule was completely demyelinated in its anterior limb and very pale in the anterior third of the posterior limb, but some rather thin fibres ran vertically in the posterior limb. Some of these were probably afferents from the thalamus. The fibres passing across the internal capsule to the putamen and globus pallidus on the other hand were quite darkly stained. The fibres in the outer part of the thalamus and on its inner border were also well-stained, but 
there was general pallor of the internal nucleus of the thalamus. In the brainstem all the tracts with the exception of the pes pedunculi and pyramidal tracts were well stained. In the pes the pyramidal tract appeared as a pale grey wedge-shaped area in the central half; this contained numerous fine vertical fibres. In the inner and outer quarters of the pes no fibres were seen except those which passed across it from the basal ganglia. In the lower part of the pons and in the medulla the pyramidal tracts contained fairly numerous rather thicker fibres, many of which had an oblique course, apparently passing dorsally as they descended. The myelin sheaths in the pyramidal tracts were definitely thicker in the medulla than in the midbrain and internal capsule. The optic nerves stained somewhat palely, but under

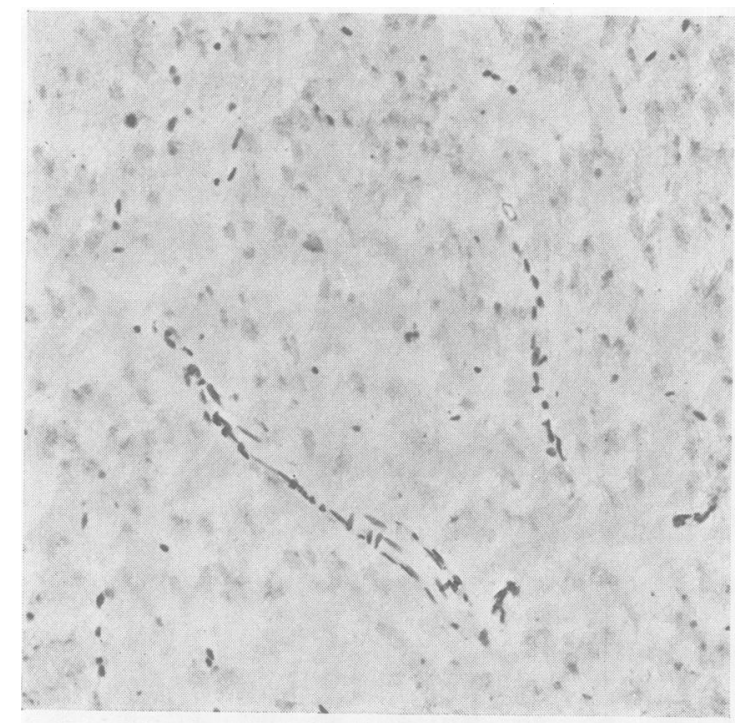

Fig. 2.-White matter of centrum ovale. The only nuclei seen are those round small vessels and isolated nuclei of astrocytes and microglia. Masses of lipoid and mucinoid material are faintly stained. Iron-hæmatoxylin, van Gieson (celloidin section).

the microscope were seen to contain many fairly thick myelin sheaths, many of which were swollen locally with balls of myelin.

In the cerebellum there was complete demyelination of the white matter both in the centre and in the folia over an area corresponding roughly to the lower half.

In sections stained for neuroglia by Mallory's phosphotungstic acidhæmatoxylin and by Anderson's Victoria blue method a great increase in the number of fibres was found, which resulted in a dense felting in the white matter of the hemispheres and in a general isomorphic increase of 
fibres in the brainstem. None was seen in the cerebral cortex or the lenticular and caudate nuclei, except just under the pial surface and the ventricular wall, but in the molecular layer of the cerebellum in its lower half the radial fibres were more distinct and thicker than normal. This neuroglial increase was thus seen not only in demyelinated areas but also in less intense form in parts where the myelin picture was perfectly normal. It was however noteworthy that whereas in the myelinated areas the neuroglial fibres were fine and lay in their normal arrangement, in the non-myelinated areas large cells with swollen cell bodies and thick processes were abundant and a few monster cells were also seen.

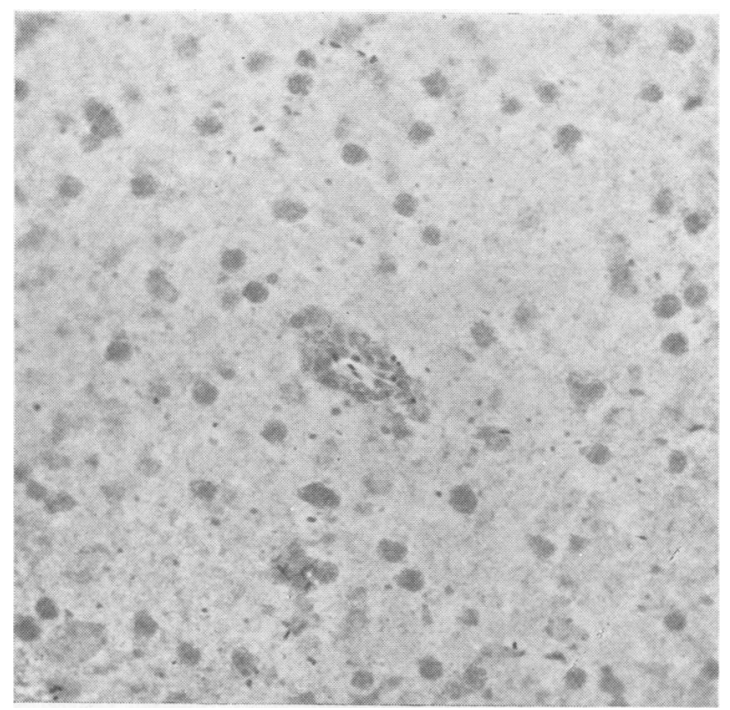

Fig. 3. White matter of centrum ovale, stained by mucicarmine and alum-hæmatoxylin. The mucinoid masses which stain fairly deeply are scattered through the tissues and distend the sheath of a small vessel. The lipoid masses do not stain. Note that the great majority of the mucinoid masses are devoid of nuclei.

In sections stained with hæmatoxylin two striking abnormalities were noted. The first was the complete absence of the small round nuclei of the interfascicular glia cells from large areas of the brain. In fact no cells of this type could be identified with certainty. In the centrum ovale no nuclei could be seen except sparse pale oval nuclei of astrocytes, and irregularlyshaped microglial and endothelial nuclei round the vessels. A similarly deserted appearance was noted in the white matter of the cerebellum. This abnormality was not confined to the demyelinated areas, for the myelinated bundles traversing the globus pallidus, in which interfascicular glial nuclei 
are normally abundant, were completely devoid of such nuclei; nor were any seen in the hilum of the inferior olives, although the fibres here also were fairly well myelinated.

The other striking feature was the presence of rounded or oval masses about $20 \mu$ in diameter which stained fairly darkly with hæmatoxylin in frozen and celloidin sections. These were thickly scattered in the demyelinated areas, where they also distended the Virchow-Robin spaces of many of the vessels. They were most abundant, and stained most deeply in a zone which lay about $2 \mathrm{~mm}$. from the cortex. The majority were completely devoid of nuclei, but in a few a minute granule which stained deeply with hæmatoxylin was seen. This may have represented a pyknotic or degenerated nucleus. With mucicarmine and metanil yellow the majority stained rather bright red, and with Heidenhain's Azan stain and Mallory's connective-tissue stain, stained fairly darkly with anilin blue, indicating their mucinoid character. But with the Azan stain there were also numerous similar masses which stained bright red, and in some both blue and red granules were seen. It was noted that in a zone a millimetre wide under the cortex the majority of the masses was of these two types, whereas internal to this zone the great majority of the masses contained only blue-staining granules. Although rounded in outline they did not appear to be enclosed in a definite cell-membrane. These masses were seen not only in the white matter of the cerebral and cerebellar hemispheres but also in the demyelinated areas of the thalamus and among the thalamo-striate fibres. They were visible in the pyramidal tracts in the internal capsule, midbrain, pons and medulla, although at the lower levels they were of smaller size. In sections stained by the Bielschowsky method, the network of axis-cylinders in the frontal region was relatively intact. Only here and there was a local globular axonal swelling seen. In the occipital lobes, there was rather more degeneration of axis-cylinders, so that in some places the network was somewhat wide-meshed, and local bulbar swellings on the nerve-fibres were more numerous. But even here there was a marked contrast between the degree of demyelination and the destruction of axis-cylinders.

CaSe II. A. H. (male), age 3, was admitted to the National Hospital in January, 1932, under Dr. Collier.

His mother and father were healthy and were not blood relations. They had had four children. The first died at the age of two years and eleven months. He was a boy born at full term; he appeared normal at birth and sat up at 10 months but never walked or talked. From the time of his birth his head was large and gradually increased in size. He was seen at the National Hospital in 1923 and the diagnosis then made was that of hydrocephalus. He had no fits and was not blind.

The second child was a boy of ten and the third a girl of seven. Both of of these were kindly examined for me by Dr. Carmichael in the out-patient department and found to be perfectly normal, healthy children. The head circumference of the elder was 21 inches and of the younger $20 \frac{1}{2}$ inches. 
The patient was a full-time child. The birth was normal. He was breast fed for 10 months. He sat up and talked at the normal ages but had never walked. At the age of 12 months he had measles and was ill with bronchitis after this for three or four weeks, but recovered and at the age of 18 months could roll and crawl about the floor. When he tried to walk his legs became crossed. Otherwise he remained in good health till three months before admission to hospital, i.e., at the age of two years and nine months, when he became ill with influenza. He ate very little, slept and dozed most of the time and his head seemed heavy. Since then he had never sat up. Two weeks later his speech became indistinct, and after a further two weeks he ceased talking altogether. For the last six weeks his mother had noticed that when he cried he became stiff; but there was no loss of consciousness or fits at any time, nor any vomiting. The child ate and slept well but had been increasingly irritable. The mother thought that he was still bright and intelligent and that his sight and hearing were unaffected. His head was said to have been of normal size at birth but to have grown large gradually.

He was found on examination to be a thin small child with a large head. He lay quietly on his back but every 10 minutes or so he appeared to have an attack of pain, his back became arched and his head retracted and he gave a sharp cry. At first all his limbs were rigidly extended, but soon the left arm was flexed and drawn over to the right shoulder. The examination of his eyes showed that the pupils reacted slowly to light. The fundus oculi was normal except for slight fullness of the retinal veins. The corneal reflexes were present, but he did not respond to pinpricks on the face, trunk or limbs. His limbs were held rigid, the left arm and the legs extended and the right arm flexed at the elbow and fingers. The tendon jerks were present but rather feeble, the abdominal reflexes were absent and the plantars of the Babinski type.

A lumbar puncture was performed on January 7. The cerebrospinal Huid was under low pressure $(96 \mathrm{~mm}$.). The fluid was clear and colourless. It contained $120 \mathrm{~mm}$. of protein per 100 c.c. and gave a positive Pandy and a weakly positive Nonne-Apelt reaction. No cells were seen. $\mathrm{He}$ died the same evening.

\section{PATHOLOGY.}

A post-mortem examination was made by me on the following day. There was comparatively little thinning of the skull; the membranes covering the brain were thin and the foramina of Magendie and Luschka open. There was no evidence of hydrocephalus or of thrombosis of the venous sinuses. The convolutions of the brain were rather more prominent than normal and the cerebral hemispheres felt very firm, almost hard, whereas the pons and cerebellum had the normal consistency. The spinal cord was removed. It appeared normal. The left lung was adherent to the left side of the chest and to the diaphragm. Otherwise the lungs appeared normal. The other organs of the body showed no abnormality. The posterior half of each eye was removed, 20 per cent. formalin having been injected into each overnight. The brain weighed 1,287 grammes; the right hemisphere, $580 \mathrm{grm}$. , the left 575 grm., and the brainstem and cerebellum 132 grammes. The relation of 
the weight of the cerebral hemispheres to that of the brainstem and cerebellum was therefore as $\mathbf{8 . 7 5}$ is to 1 , the normal, according to Dunlap, being in the adult as 7 to 1 .

The microscopic appearances in this case were so similar to those in Case I that it is only necessary to indicate the details in which they differed. In this case the degeneration was less advanced, in that throughout the white matter of the hemispheres there was a network of fine myelinated fibres interspersed with many myelin balls. Thus to the naked eye the sections appeared pale grey. On the whole there was more demyelination in the occipital than in the frontal lobes, but the difference was not great. The optic radiations stood out as a more darkly stained band, but here also some myelin balls could be seen under the microscope. The appearances of the basal ganglia were the same, except that there was less shrinkage and demyelination of the inner nucleus of the thalamus. In the midbrain the appearance was almost exactly similar to that in Case I, in that while the pyramidal tracts stained faintly the frontopontine and occipital fibres in the crura were unstained. In the cerebellum all the white centre, including the stems of the folia, was fairly well myelinated, although it stained less deeply in the lower than in the upper half. In the cord the pyramidal tracts were pale and the sides of the cord were shrunken in this region, but otherwise there was no abnormality. The optic nerves as a whole stained fairly dark, but on microscopic examination the fibres were rather isolated from one another and irregular in calibre, and balls of myelin could be seen here also. With hæmatoxylin alone similar masses of what appeared to be lipoid material could be seen. This certainly stained less intensely after 48 hours in such fat solvents as xylol, chloroform and a mixture of absolute alcohol and ether, but it did not disappear completely. In sections stained with Scharlach $\mathbf{R}$ and alum-hæmatoxylin many of the vessels in the white matter of the cerebral and cerebellar hemispheres were ringed with a layer of granule-cells with bright red fatty inclusions, but no brightly staining lipoid could be seen outside the perivascular sheaths. The dark masses which were seen in sections stained with iron-hæmatoxylin only, appeared after Scharlach staining as pale lavender-coloured bodies. Only a certain proportion of these stained with mucicarmine, but in certain situations, as for example, near the dentate nucleus of the cerebellum and in areas in the occipital white matter, mucinoid masses similar to those in Case I were fairly abundant. With Heidenhain's Azan stain also the picture was slightly different from that in Case I, in that a larger proportion of the masses stained red and a smaller proportion blue. The disappearance of interfascicular oligodendroglia nuclei also was not so complete as in Case I. They were normally present in the spinal cord, and here no mucinoid or lipoid globules were seen. Degenerated nuclei of this kind were also seen in the less affected fibre-systems, for example in parts of the cerebellar hemispheres, in the hilus of the olives, 
the reticular formation and transverse fibres of the pons and among the thalamo-striate fibres in the basal ganglia. With Bielschowsky's method a network of fine fibres was seen throughout the white matter of the cerebral hemispheres, in fact there appeared to be little destruction of axis-cylinders. The retinæ were normal.

The changes were thus less pronounced than in Case $I$ and indicated a rather earlier stage in a similar degenerative process. This was brought out especially well with Heidenhain's Azan stain, by which it appeared that the earlier stages of lipoid degeneration were stained red and the later stages blue. Thus in the pons the globules among the pyramidal fibres were chiefly blue and those among the transverse fibres of the pons mostly red. Similarly in Case I the large proportion of red-staining granules in the subcortical zone may be taken as evidence that this zone was last affected.

There was also rather less intense neuroglial overgrowth in this case than in Case I, although in the cerebellum, in spite of the relative preservation of the myelin sheaths, there was a considerable degree of neuroglial sclerosis which affected the molecular layer to an almost equal degree to that seen in Case I. Another interesting observation in the cerebellar cortex in both cases was the paucity of basket-fibres. Otherwise the cell and fibre structure of the cerebellar cortex was well preserved.

\section{DISGUSSION.}

These two cases are thus so similar in their clinical and pathological pictures that there can be no doubt that they are two examples of one diseaseprocess. It is equally clear that this process differs definitely and perhaps essentially from that of Schilder's disease. In fact it is only in the rarefaction of myelin sheaths in the centrum ovale and the cerebellar hemispheres and in the compensatory gliosis that the two conditions resemble one another. The condition described approximates closely both in distribution and degree of demyelination to the state of the cerebral hemispheres in a three-months baby. This correspondence is seen not only in the striking contrast of the well-myelinated optic radiations to the unmyelinated areas round them, but also in the state of myelination of the basal ganglia and the pes pedunculi. It is noteworthy also that the lack of myelin in the hemispheres is associated with a fairly well preserved network of axis-cylinders; and the comparative thickness of the myelin sheaths of the pyramidal tracts in the medulla as compared with those in the internal capsule suggests a process of local malnutrition of myelin.

The other striking features about this condition are:-

(1) The diffuse nature of the gliosis, which was noticeable in regions where the myelination was intact although less pronounced here than in the white centres of the cerebrum and cerebellum. 
(2) The complete or almost complete absence of nuclei of the interfascicular glia, not only from the demyelinated tracts but also from some of those in which the myelination was still fairly normal.

(3) The presence in enormous numbers of balls of mucinoid and abnormal lipoid material.

In association with these features there was a remarkably slight cellular reaction in the demyelinated areas so that fat-granule cells were only to be seen in the Virchow-Robin spaces. This suggests that no very active breaking down of preexisting myelin was taking place.

The chief problem in these cases is to explain the distribution of the demyelination in relation to the absence of interfascicular glial cells. These cells had obviously degenerated first and the demyelination of fibre-systems appeared to be secondary to this degeneration. Along with their degeneration there was a deposition first of lipoid and later of mucinoid material, which eventually found its way into the Virchow-Robin spaces. To some extent this material might have been derived from the degeneration of the interfascicular oligodendrocytes, although typical ' mucocytes' of Grynfeltt were absent in Case I and very scarce in Case II.

It is specially noteworthy that the demyelination affected only those tracts in which myelination does not occur till after birth, and was most marked in those systems in which myelination is delayed until after the third month. It is well known that the differentiation of interfascicular oligodendroglia goes hand in hand with myelination. Thus at birth there are numerous oligodendroblasts in the unmyelinated systems but very few mature oligodendrocytes. The explanation of these two cases may therefore lie in a failure of differentiation of oligodendroglia cells and in a greater vulnerability to febrile and metabolic disturbances of these undifferentiated cells. On the other hand the abundant masses of abnormal lipoid and mucinoid material recall the suggestion of Bielschowsky and Henneberg, who also found masses of abnormal lipoid in their cases, that the material from which the myelin is formed might be supplied in abnormal form. The thinness and pallor of the myelin sheaths in the cerebral hemispheres and the absence of any reactive fat phagocytosis, certainly suggested rather a failure of nutrition of myelin sheaths than an active destruction of myelin. It is possible that during fotal life the nutritive material was normally supplied from the maternal blood, so that up till birth myelination proceeded along normal lines; and after birth this substance may have been to a limited extent available from the mother's milk, so that symptoms did not show themselves until this supply was withdrawn.

In spite of great differences there are certain affinities between these cases and the familial cases of Schilder's disease. In the cases of the latter kind that I have had the opportunity of examining it was noticeable that one of the earliest alterations in the affected areas was a loss of anisotropic 
power by the myelin, and that anisotropic granules were very scarce in the fat phagocytes. In the two cases here described there was a similar lack of anisotropic properties. The thickest myelin sheaths were indeed doubly refractile in a faint manner, but no brightly anisotropic material was seen anywhere in the affected areas.

It was noted by Collier and Greenfield that the disease-process in their familial case ran along fibre-systems such as the pyramidal tracts and the anterior commissure. Among the fibres of these systems the abnormal cells called by them ' globoid cells' were abundant, although no such cells could be seen in neighbouring unaffected areas.* In the two cases here described there was also an affection of fibre-systems, although the histological picture in the affected systems bore no resemblance to that described by Collier and Greenfield. Here one must again insist on the fact that mucinoid globules were found among the pyramidal tract fibres throughout the brain-stem, but not (in Case II at any rate) in the spinal cord. This fact at once distinguishes the degeneration of the pyramidal tract in these cases from Wallerian degeneration due to a lesion in the white matter of the hemispheres.

Whether these cases were familial is not at present definitely known. The history of the eldest child in the family of Case II was so similar to that of our patient that it appears probable that he was suffering from the same condition. But it is possible that the diagnosis of hydrocephalus in his case was the correct one. As Case I was a first born and only child no evidence of familial incidence is here available. In any case the condition appears to be of a purely degenerative character and to be in some degree at least a developmental error revealing itself in the early years of life.

\section{SUMMARY.}

1. Two cases of cerebral sclerosis occurring in young children are described. Both patients died at the age of three years; in one case there was some indication that a previous member of the family had been similarly affected.

2. The demyelination in these cases affected the white matter of the cerebral hemisphere diffusely, but the optic radiations and the pyramidal tracts were relatively spared. The fibres which myelinate latest appeared to be primarily affected.

3. The interfascicular oligodendrocytes were absent in the case with the longer history, and very degenerated in that with the shorter history. Along with this there was a remarkable accumulation of masses of mucinoid

* N.B.-These cells differed in many respects from the normal reacting types of neuroglial cell (gemästete Gliazell) although we considered that they were of neuroglial origin. Prof. Spielmeyer, however, who kindly examined our sections, thought that they were of mesodermal origin, and Carnegie Dickson, who has found similar cells in the younger members of the same family, is of a similar opinion. 
and abnormal lipoid granules. These were found throughout the pyramidal tract in the brainstem and in other partially demyelinated fibre-systems as well as in the most demyelinated areas.

4. The pathogenesis of the condition appears to be failure of nutrition of the myelin sheaths owing to the degeneration of the interfascicular glia. The latter may be due to faulty development of those oligodendrocytes which are differentiated last.

5. It is suggested that the primary cause of the condition may be the child's inability to form the proper lipoid material for the formation of myelin.

It is a pleasure to thank Drs. Collier and Critchley for their permission to publish these cases and Professor Lucas Keene and Dr. Evelyn Hewer for valuable assistance on the myelination of the developing human brain.

\section{REFERENCES.}

1 Berliner, M. L., Arch. of Ophthalmol., 1931, vi, 740.

2 Biflschowsky, M., and Henneberg, R., Jour. f. Psychol. u. Neurol., 1928, xxxvi, 131 .

${ }^{3}$ Coenen, L., and Mir, L., L'Encéphale, 1931, xxvi, 357.

4 Collier, J., and Greenfield, J. G., Brain, 1924, xlvii, 489.

5 Del Rio Hortega, Memorias de la Real Sociedad Espaniola de Historia Naturale, 1928, xiv, 7.

${ }^{6}$ Grynfeltt, E., C. R. soc. de biol., 1923, Ixxxix, 1264.

7 KrabBe, K., Brain, 1916, xxxix, 74.

${ }^{8}$ Levaditi, C., Ann. de l'Institut Pasteur, 1930, xlv, 673.

9 Marinesco, G., Jour. f. Psychol. u. Neurol., 1930, xli, 1.

10 Merzbacher, L., Zeits. f. d. g. Neurol. u. Psychiat., 1910, iii, 1.

11 Pelizaeus, Archiv f. Psychiat., 1885, xvi, 678.

12 Scholz, W., Zeits. $f$. d. g. Neurol. u. Psychiat., 1925, хcix, 651.

13 SJögren, Torste, Die juvenile amaurotische Idiotie, 1931. 